\title{
Effectiveness of a Formal Mentorship Program in Family Medicine Residency: The Residents' Perspective
}

\author{
Marie Andrades, ${ }^{1}$ Seema Bhanji, ${ }^{1}$ Mariam Valliani, ${ }^{1}$ \\ Foaud Majeed, ${ }^{1}$ and Sheilla Kumari Pinjani ${ }^{2}$ \\ ${ }^{1}$ Family Medicine Department, Aga Khan University, Stadium Road, P.O. Box 3500, Karachi 74800, Pakistan \\ ${ }^{2}$ Department for Educational Development, Aga Khan University, Stadium Road, P.O. Box 3500, Karachi 74800, Pakistan
}

Correspondence should be addressed to Seema Bhanji; seema.bhanji@aku.edu

Received 6 December 2012; Accepted 21 January 2013

Academic Editor: Balakrishnan Nair

Copyright (C) 2013 Marie Andrades et al. This is an open access article distributed under the Creative Commons Attribution License, which permits unrestricted use, distribution, and reproduction in any medium, provided the original work is properly cited.

Introduction. Mentoring is a recognized form of teaching learning strategy in postgraduate medical education. This paper describes the effectiveness of a formal mentorship program from the residents' perspective after a year of implementation.Methods. The Aga Khan University Family Medicine Residency Program is the first program in Pakistan to our knowledge to implement formal mentorship for all four years of residency. A mentorship program was developed, implemented, and evaluated a year later using a rating scale. The 10-point Likert scale consisted of questions on academics, clinical work, research, administrative issues, and personal/social issues. Results. The response rate was 95\% $(n=20)$. Eighty percent $(n=16)$ were women. Satisfaction level in seeking help was the highest for academics (75\%). Residents scored mentorship as low in helping to tackle their personal problems (20\%). Barriers reported in rapport building with mentor were time constraints and gender difference. The most useful attributes of the mentor which helped rapport building were accessibility, active listening, support for emotional needs, and trustworthiness. Conclusion. Mentoring has a role in trainees' personal and professional growth especially when their needs are addressed. The effectiveness of the mentorship program in residency can improve if the residents are allowed to choose their own mentors.

\section{Introduction}

Mentoring is a recognized form of teaching learning strategy in postgraduate medical education. Mentoring programs appear to play an essential role in creating a learner-centered environment for fostering professionalism and humanistic values [1]. Through mentoring, faculty shares their special skills and experience with junior colleagues [2]. It creates an environment where sensitive issues can be openly raised for reflective constructive discussions. It is considered as a mean of identifying and managing professional and personal progress with minimal stress. Physicians with no obvious difficulties also benefit in terms of academics, clinical skills, and future planning [3].

The Aga Khan University Family Medicine Residency Program had no formal mentorship program for residents. Each resident has a supervisor approved by the College of Physicians and Surgeons Pakistan, the accrediting body for postgraduate training in Pakistan. Supervisor's role is more of a teacher and an appraiser than a mentor. The residents and the residency management committee felt the need for a formal mentorship program wherein residents could share their personal and professional problems. Based on this need a formal mentorship program was introduced in November 2007.

The objective of this study was to describe the effectiveness of mentorship program from residents' perspective after a year of implementing a formal mentorship program in the family medicine residency.

\section{Methods}

The Aga Khan University Family Medicine Residency Program is a four-year program with a total of six residents per year. At the time of the intervention there were 21 residents in the program. 
The residency committee, comprising of three faculty and two senior residents, developed a mentorship plan. A one-to-one mentoring model using the traditional senioritybased mentoring approach was used, as it best suited the stage of development of the residents and was suggested by them [4]. A total of eight faculty expressed willingness to be involved in the program. An initial meeting was held with the residents and the faculty to discuss the responsibilities of the residents and the faculty as mentees and mentors, respectively. A mutually agreed definition for mentorship was developed based on internationally accepted definitions and faculty and residents views on mentorship along with roles and responsibilities of the mentor and mentee. Mentors agreed to encourage mentee to discuss issues related to work, education, personal development, and psychosocial aspects [5]. Confidentiality of the matters discussed in meetings was agreed upon by both parties, unless it was imperative for the mentor to disclose information for the benefit of a mentee or if mentee perceived that she/he needed a second opinion. Serious issues outside the domain of resolution by the mentor were decided to be brought to the residency committee with the permission of the mentee.

The faculty mentors were assigned to the residents by the committee for a period of one year after which the relations could be reviewed. Residents were given a choice to change their mentor if they desired so; however, none did so.

A year after the intervention, the residency committee evaluated the effectiveness of the mentorship program from the residents' perspective. For this purpose a questionnaire was developed based on the literature [6-8], consisting of questions regarding assistance provided by the mentor in areas of clinical work, academics, research, administration and personal development. These were assessed on a 10-point Likert scale from strongly agree as 10 to strongly disagree as 1. Residents were also required to rank the three most useful attributes of their mentor they found most helpful (from a list of attributes provided) and report on the frequency of meetings between the mentor and mentee.

Reasons for seeking help and perceived barriers to the relationship were elicited through open ended responses.

\section{Findings}

We had a response rate of $95 \%(n=20)$. One resident was out of station and was unable to complete the questionnaire. Eighty percent of residents were women $(n=16)$, and twenty percent were men $(n=4)$. Amongst eight faculty mentors, two were men.

A quarter of the residents were able to meet their mentors every month, $25 \%$ could meet every quarter, and $50 \%$ met their mentors less frequently.

Majority (85\%) of the times, the meetings were initiated by the mentee.

The main areas for which residents sought help as seen in Figure 1 were problem solving and research. Satisfaction level in seeking help was the highest for academics (75\%). Twenty percent of the residents did not feel comfortable discussing personal problems (residents reported time constraints and

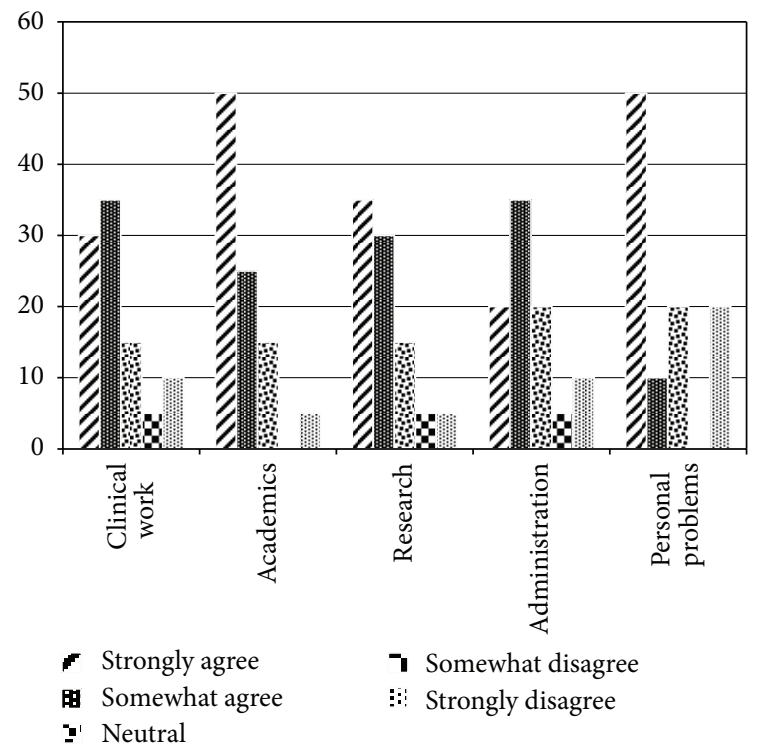

FIGURE 1: Residents' opinion on aspects in which mentorship helped (\%).

gender difference as the major barriers to a successful mentoring relationship). The attributes mentees found most helpful in the mentoring relationship were accessibility, active listening, support for emotional and psychological needs, and trust in relationship.

\section{Discussion}

This study adds to the understanding of the advantages and barriers to a formal mentoring program. This study supports the role of mentoring in trainees' professional growth especially when their needs are addressed. This study is similar to the one conducted for orthopedic residents where the residents were most satisfied with the mentoring when a formal program was in place. Nearly all the residents in this study placed a high value on the mentorship program and felt that the mentors were beneficial to their training especially for academics and clinical input [7].

In our study, addressing personal problems were scored as the area of least satisfaction by resident mentees perhaps due to the gender difference between mentor and mentee and the heavy service component of both mentors and mentees leading to limited accessibility.

The barriers to mentorship reported were lack of time, gender difference between mentor and mentee, and lack of rapport which is also reflected in the study by Williams et al. [8]. Lack of time from the mentor could be due to absence of protected time and the faculty training as mentors. Though faculty volunteered for mentorship and were motivated, the fact that there was no compensation in terms of revenue or academic recognition could have been a deterrent to their motivation, Flint et al. [7] had similar observations. Gender difference between mentor and mentee can prevent an effective relationship particularly in Asian Pakistani culture. Men may be less able to provide guidance on the needs of female 
mentees especially in work and life balance [9], and it would be difficult for a woman to discuss personal issues particularly related to family life like relationship with in-laws/husband especially in the South Asian culture where women are not expected to discuss such issues with male colleagues. It is therefore important to provide a good match between mentor and mentee both in personality traits and chemistry which leads to a helpful relationship [10].

The study has limitations. The small sample size may make it difficult to generalize conclusions to wider context. However, the sample did consist of the entire population of the residents except one.

\section{Conclusion}

A formal mentorship program does help residents in their personal and professional lives. Faculty development would enhance the program. To make it more effective, residents should be allowed to choose their own mentors, and protected time should be provided to residents and faculty. Faculty contributing quality time and effort ought to be acknowledged academically through rewards such as recognition during the promotion process.

\section{Conflict of Interests}

The authors declare to have no any conflict of interests.

\section{Acknowledgments}

The Authors wish to thank the family medicine residents who participated and gave their feedback and the faculty mentors who were forthcoming with their time and advice.

\section{References}

[1] S. J. Lieff and D. Clarke, "What factors contribute to senior psychiatry residents' interest in geriatric psychiatry? A delphi study," Canadian Journal of Psychiatry, vol. 45, no. 10, pp. 912916, 2000

[2] P. A. Setness, "Mentoring: leaving a legacy of opportunity and responsibility," Postgraduate Medicine, vol. 100, no. 4, pp. 15-22, 1996.

[3] R. Alliot, "Facilitatory mentoring in general practice," British Medical Journal, vol. 313, no. 7060, p. 2, 1996.

[4] B. Buddeberg-Fischer and K. D. Herta, "Formal mentoring programmes for medical students and doctors-a review of the Medline literature," Medical Teacher, vol. 28, no. 3, pp. 248-257, 2006.

[5] K. M. Markakis, H. B. Beckman, A. L. Suchman, and R. M. Frankel, "The path to professionalism: cultivating humanistic values and attitudes in residency training," Academic Medicine, vol. 75, no. 2, pp. 141-150, 2000.

[6] R. A. Berk, J. Berg, R. Mortimer, B. Walton-Moss, and T. P. Yeo, "Measuring the effectiveness of faculty mentoring relationships," Academic Medicine, vol. 80, no. 1, pp. 66-71, 2005.

[7] J. H. Flint, A. A. Jahangir, B. D. Browner, and S. Mehta, "The value of mentorship in orthopaedic surgery resident education: the residents' perspective," Journal of Bone and Joint Surgery A, vol. 91, no. 4, pp. 1017-1022, 2009.
[8] L. L. Williams, J. B. Levine, S. Malhotra, and P. Holtzheimer, "The good-enough mentoring relationship," Academic Psychiatry, vol. 28, no. 2, pp. 111-115, 2004.

[9] K. Leslie, L. Lingard, and S. Whyte, "Junior faculty experiences with informal mentoring," Medical Teacher, vol. 27, no. 8, pp. 693-698, 2005.

[10] V. A. Jackson, A. Palepu, L. Szalacha, C. Caswell, P. L. Carr, and T. Inui, "Having the right chemistry': a qualitative study of mentoring in academic medicine," Academic Medicine, vol. 78, no. 3, pp. 328-334, 2003. 

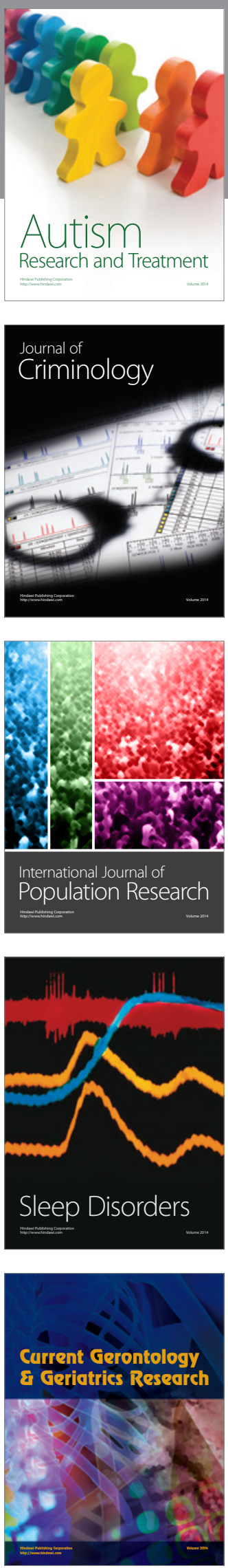
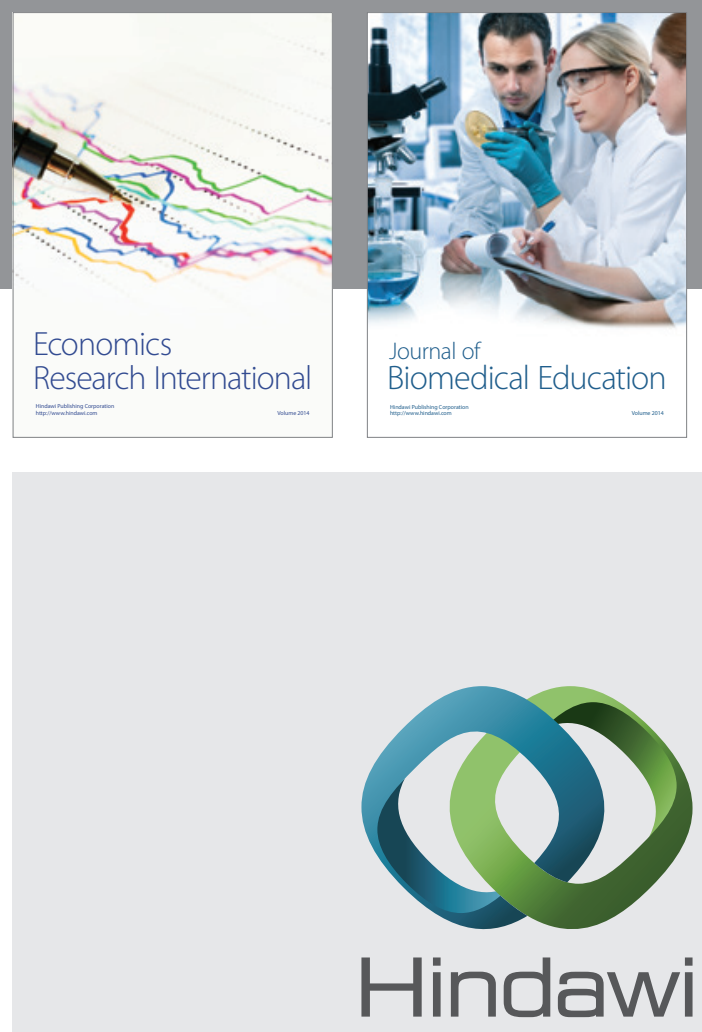

Submit your manuscripts at

http://www.hindawi.com
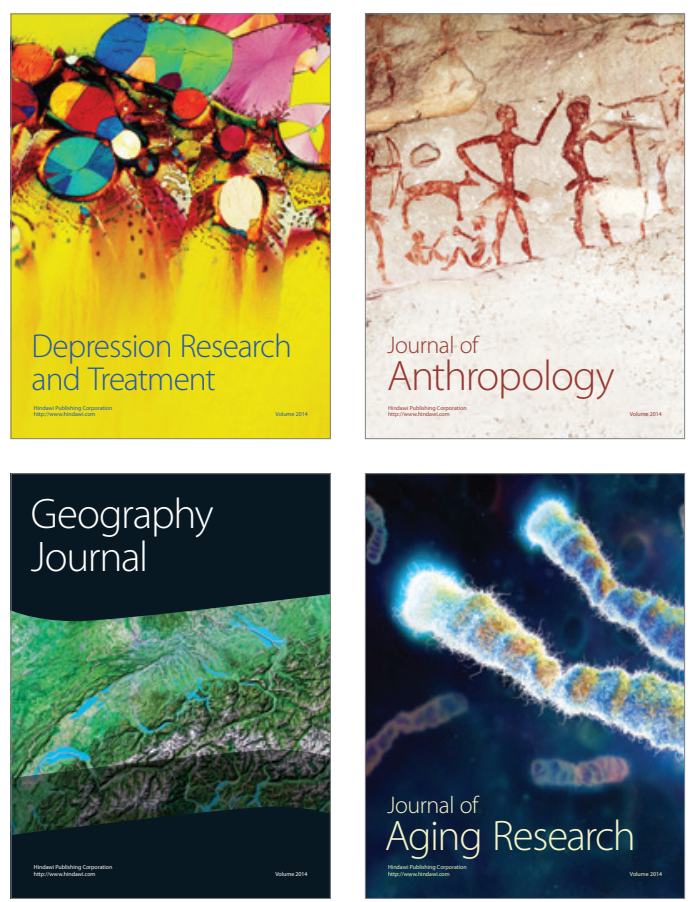
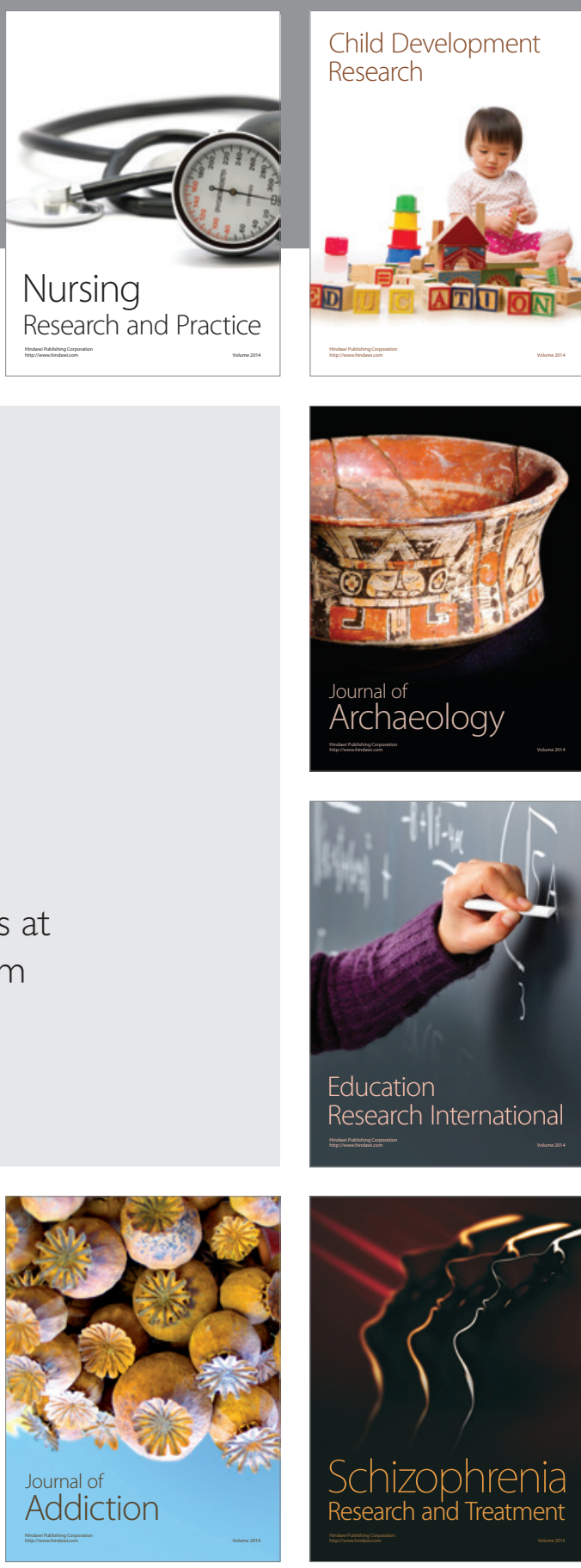

(D)
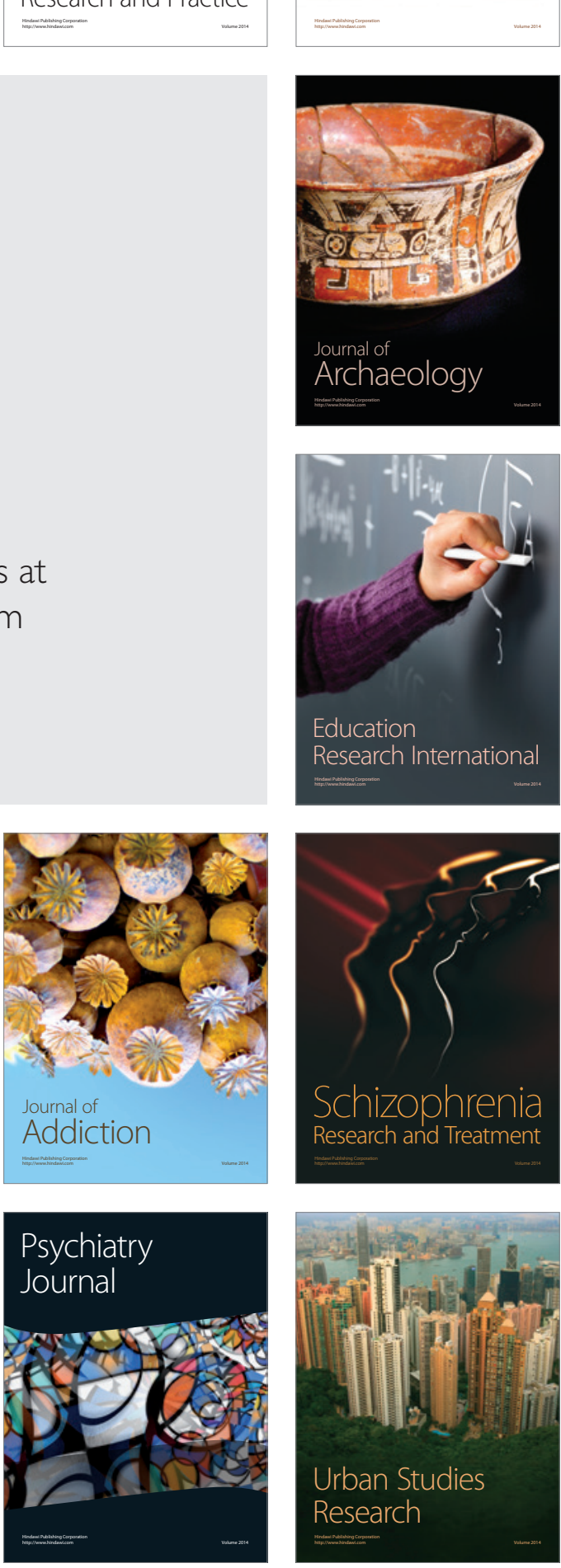